\title{
Method of intermediate problems in the Fröhlich polaron model
}

\author{
A.V. Soldatov* \\ V.A. Steklov Mathematical Institute, Department of Mechanics, 8 Gubkina Str., 119991 Moscow, Russia
}

Received June 13, 2009, in final form July 14, 2209

\begin{abstract}
Method of intermediate problems in the theory of linear semi-bounded self-adjoint operators on rigged Hilbert space was applied to the investigation of the ground state energy of the Fröhlich polaron model. It was shown that various infinite sequences of non-decreasing improvable lower bound estimates for the polaron ground state energy can be derived for arbitrary values of the electron-phonon interaction constant. The proposed approach allows for explicit numerical evaluation of the thus obtained lower bound estimates at all orders and can be straightforwardly generalized for investigation of the low-lying branch of the slow-moving polaron excitation energy spectral curve adjacent to the ground state energy of the polaron at rest. In conjunction with numerous, already derived by multitudinous methods, well-known upper bound estimates for the energy spectral curve of the Fröhlich polaron as a function of the electron-phonon interaction constant and the polaron total momentum, the aforesaid improvable lower bound estimates might provide one with virtually precise magnitude for the energy of the slow-moving polaron.
\end{abstract}

Key words: polaron, Fröhlich polaron model, lower bound estimates, method of intermediate problems, ground state energy

PACS: $71.38 .-k, 71.38 . F p$

\section{The polaron concept and the Fröhlich polaron model}

It is well known that a local change in the electronic state in a crystal leads to the excitation of crystal lattice vibrations, i. e. the excitation of phonons. And vice versa, any local change in the state of the lattice ions alters the local electronic state. This situation is commonly referred to as an "electron-phonon interaction". This interaction manifests itself even at the absolute zero of temperature, and results in a number of specific microscopic and macroscopic phenomena such as, for example, lattice polarization. When a conduction electron with band mass $m$ moves through the crystal, this state of polarization can move together with it. This combined quantum state of the moving electron and the accompanying polarization may be considered as a quasiparticle with its own particular characteristics, such as effective mass, total momentum, energy, and maybe other quantum numbers describing the internal state of the quasiparticle in the presence of an external magnetic field or in the case of a very strong lattice polarization that causes self-localization of the electron in the polarization well with the appearance of discrete energy levels. Such a quasiparticle is usually called a "polaron state" or simply a "polaron". Hence, polaron formation is a consequence of dynamic electron-lattice interaction.

The concept of the polaron was introduced first by L.D. Landau in a very short paper [1], followed by much more detailed work by S.I. Pekar [2] who investigated the most essential properties of stationary polaron in the limiting case of very intense electron-phonon interaction, so that the polaron behavior could be analyzed in the so-called adiabatic approximation. Subsequently, Landau and Pekar [3] investigated the self-energy and the effective mass of the polaron for the adiabatic or strong-coupling regime. Many other famous researchers, among them H. Fröhlich, R. Feynman and N.N. Bogolyubov, contributed to the development of polaron theory later [4-6,8-10]. Since its inception, the polaron concept remains of interest from at least two points of view, practical

*E-mail: soldatov@mi.ras.ru 
and theoretical as well: it describes the physical properties of charge carriers in polar crystals and ionic semiconductors and, at the same time, represents a simple but rich in content field-theoretical model of a particle interacting with a scalar boson field.

The model under consideration is the standard quantized Fröhlich polaron Hamiltonian introduced by H. Fröhlich [6]

$$
H=\frac{\hat{\mathbf{p}}^{2}}{2 m}+\hbar \omega \sum_{\mathbf{k}} a_{\mathbf{k}}^{+} a_{\mathbf{k}}+\sum_{\mathbf{k}}\left(V^{*}(k) a_{\mathbf{k}}^{+} \mathrm{e}^{-\mathrm{i} \mathbf{k} \hat{\mathbf{r}}}+\text { h.c. }\right),
$$

where

$$
V(k)=-\mathrm{i} \frac{\hbar \omega}{k}\left(\frac{4 \pi \alpha}{V} \sqrt{\frac{\hbar}{2 m \omega}}\right)^{1 / 2} .
$$

The operators $\hat{\mathbf{p}}$ and $\hat{\mathbf{r}}$ stand for the electron momentum and position coordinate quantum operators, satisfying the usual commutation relations

$$
\left[\hat{p}_{i}, \hat{r}_{j}\right]=-\mathrm{i} \hbar \delta_{i j}
$$

and the operators $a_{\mathbf{k}}^{+}, a_{\mathbf{k}}$, satisfying the usual commutation relations

$$
\left[a_{\mathbf{k}}, a_{\mathbf{k}^{\prime}}^{+}\right]=\delta_{\mathbf{k k}^{\prime}}, \quad\left[a_{\mathbf{k}}, a_{\mathbf{k}^{\prime}}\right]=0,
$$

are Bose operators of creation and annihilation of longitudinal optical phonons of energy $\hbar \omega$ and wave vector $\mathbf{k}$. It is assumed that the phonon wave vector runs over a very large but finite quasidiscrete set of values

$$
\mathbf{k}=\left\{\frac{2 \pi}{L a} n_{1}, \frac{2 \pi}{L a} n_{2}, \frac{2 \pi}{L a} n_{3}\right\}, \quad n_{i}=0, \pm 1, \pm 2, \ldots, \pm(L / 2-1),+L / 2, \quad i=1,2,3
$$

where $a^{3}$ is the volume of the unit crystal cell and $L^{3}$ is the number of these cells within the volume $V$ of the crystal, $L$ assumed to be even. The limit $V \rightarrow \infty$ corresponds to the rule of the transition from the quasi-discrete to continuous spectrum

$$
\lim _{V \rightarrow \infty} \frac{1}{V} \sum_{\mathbf{k}} \cdots \rightarrow \frac{1}{(2 \pi)^{3}} \int \mathrm{d} \mathbf{k} \cdots=\frac{1}{(2 \pi)^{3}} \int_{0}^{2 \pi} \mathrm{d} \phi \int_{0}^{\pi} \mathrm{d} \theta \sin \theta \int_{0}^{k_{\mathrm{D}}} \mathrm{d} k k^{2} \ldots
$$

to be applied to all relevant expressions. Here $k_{\mathrm{D}}=\left(6 \pi^{2}\right)^{1 / 3} / a$ is the Debye wave vector, $a$ being the lattice constant. For any realistic, or "physical", observable polaron, the value of $k_{\mathrm{D}}$ is finite whilst the limit $k_{\mathrm{D}} \rightarrow \infty$ corresponds to the so-called "field-theoretical" polaron model. It is important to emphasize from the beginning that in this study we are preoccupied mainly with physical polaron model. Extensive useful discussion on various aspects of phenomenological polaron physics as well as on the derivation of physical quantum polaron model (1) and methods of its treatment can be found in [7] and references therein. For the matter of convenience it is assumed further on that $\hbar=\omega=m=1$.

\section{Low-lying branch of the polaron energy spectrum}

It is known that the polaron total momentum

$$
\hat{\mathbf{P}}=\hat{\mathbf{p}}+\sum_{\mathbf{k}} \mathbf{k} a_{\mathbf{k}}^{+} a_{\mathbf{k}}
$$

is a constant of the motion and commutes with the Hamiltonian (1). Therefore, it is possible to transform the Hamiltonian to the representation in which $\hat{\mathbf{P}}$ becomes a "c"-number by means of the unitary transformation

$$
H \rightarrow \tilde{H}, \quad \tilde{H}=S^{-1} H S, \quad S=\exp \left(-\mathrm{i} \sum_{\mathbf{k}} \mathbf{k r} a_{\mathbf{k}}^{+} a_{\mathbf{k}}\right)
$$


so that

$$
\tilde{H}=\frac{1}{2}\left(\hat{\mathbf{p}}-\sum_{\mathbf{k}} \mathbf{k} a_{\mathbf{k}}^{+} a_{\mathbf{k}}\right)^{2}+\sum_{\mathbf{k}} a_{\mathbf{k}}^{+} a_{\mathbf{k}}+\sum_{\mathbf{k}}\left(V^{*}(k) a_{\mathbf{k}}^{+}+h . c .\right)
$$

or

$$
\tilde{H}=\frac{1}{2}\left(\mathbf{P}-\sum_{\mathbf{k}} \mathbf{k} a_{\mathbf{k}}^{+} a_{\mathbf{k}}\right)^{2}+\sum_{\mathbf{k}} a_{\mathbf{k}}^{+} a_{\mathbf{k}}+\sum_{\mathbf{k}}\left(V^{*}(k) a_{\mathbf{k}}^{+}+h . c .\right),
$$

in the $\hat{\mathbf{p}}$ - representation where $\hat{\mathbf{P}}$ becomes a quantum "c" - number $\mathbf{P}$, the value of the polaron total momentum, and the Hamiltonian (2) no longer contains the electron coordinates. Another unitary transformation

$$
\tilde{H} \rightarrow \tilde{\tilde{H}}, \quad \tilde{\tilde{H}}=U^{-1} \tilde{H} U, \quad U=\exp \left\{-\mathrm{i} \sum_{\mathbf{k}}\left(V(k) a_{\mathbf{k}}^{+}-V^{*}(k) a_{\mathbf{k}}\right)\right\}
$$

provides us with the Hamiltonian

$$
\tilde{\tilde{H}}=\frac{1}{2}\left(\mathbf{P}-\sum_{\mathbf{k}} \mathbf{k}\left(a_{\mathbf{k}}^{+}-V(k)\right)\left(a_{\mathbf{k}}-V^{*}(k)\right)\right)^{2}+\sum_{\mathbf{k}} a_{\mathbf{k}}^{+} a_{\mathbf{k}}-\sum_{\mathbf{k}}|V(k)|^{2},
$$

which is just the sole Hamiltonian to be treated further on. The ultimate goal is to find the lowest eigenvalue $E(P)$ of this Hamiltonian for a given total polaron momentum $\mathbf{P}$. Then, for low-lying polaron energy levels the function $E(P)$ might be represented as an expansion

$$
E(P)=E_{\mathrm{g}}+\frac{P^{2}}{2 m_{\mathrm{eff}}}+O\left(P^{4}\right)
$$

where $E_{\mathrm{g}}$ is the polaron ground state energy and the coefficient $m_{\mathrm{eff}}$ can be interpreted as the polaron effective mass. Extensive work has already been done to evaluate $E(P)$ directly through conventional perturbational calculations or to find upper bound estimates for $E(P)$ by means of multitudinous variational methods. These approaches are beyond the scope of this work. It is only worth noticing that, as a rule, perturbational schemes do not provide us with reliable error bound estimates whilst the upper bounds can be relied upon only if they are supplemented with corresponding lower bounds for the magnitude in question. To our knowledge, lower bounds to the polaron energy spectrum have been receiving much less attention than the upper bounds throughout very long history of polaron studies. Among the most remarkable contributions several works by E.H. Lieb should be mentioned first of all [11,12] as well as the succeeding work by D.M. Larsen [13], who improved the result of [11], though neither of the lower bounds to the polaron ground state energy presented in these papers comes close to the best lowest upper bounds available so far. Moreover, no algorithm has been proposed to improve these bounds step by step in a regular way.

The purpose of the present research is to show that improvable lower bound estimates for the low-lying branch of the polaron energy spectrum can be derived by the method of intermediate problems in the theory of semi-bounded linear Hermitian operators on rigged Hilbert space. The principal idea of the method goes back to H. Weyl [15] and A. Weinstein [16], and has been elaborated since then by numerous contributors (see, for example, $[17,18]$ and references therein) with regard to problems of classical and quantum mechanics.

\section{Basics of the method of intermediate problems}

To make this proceeding self-contained, some technicalities of the method of intermediate problems are outlined here in brief. The starting point of the method is the standard time-independent Schrödinger equation

$$
H \psi=E \psi,
$$


where $H$ is some Hermitian operator with respect to the inner product $(\phi, \psi)=\int \phi^{*} \psi \mathrm{d} \tau$ in Hilbert space. It is assumed that all continuous spectrum energy levels of $H$ are higher than the lowest discrete spectrum energy levels of one's interest. Let us assume, too, that these discrete eigenvalues of $H$ can be ordered in a nondecreasing sequence,

$$
E_{1} \leqslant E_{2} \leqslant \ldots
$$

in which each degenerate eigenvalue, if any happens to be among others, appears the number of times of its multiplicity. Eigenstates $\psi_{i}$, corresponding to the eigenvalues $E_{i}$, satisfy the equation

$$
H \psi_{i}=E_{i} \psi_{i}
$$

and are assumed to be orthonormalized, so that

$$
\left(\psi_{i}, \psi_{j}\right)=\delta_{i j}
$$

where $\delta_{i j}$ is Kronecker's delta. It is further assumed that the Hamiltonian $H$ can be decomposed as

$$
H=H^{0}+H^{\prime}
$$

where $H^{0}$ has known eigenvalues and eigenstates and $H^{\prime}$ is an arbitrary Hermitian operator which is to be positively definite in the sense that

$$
\left(\psi, H^{\prime} \psi\right)=\int \psi^{*} H^{\prime} \psi \mathrm{d} \tau>0, \quad(\psi \neq 0)
$$

for every $\psi$ in the domain of $H$. Hereafter, it is assumed that the lowest part of the discrete spectrum of $H^{0}$ is below its continuous spectrum and that the corresponding discrete eigenvalues can be ordered in the same manner (4) as the ones belonging to the total Hamiltonian $H$

$$
E_{1}^{0} \leqslant E_{2}^{0} \leqslant \ldots,
$$

with the degenerate eigenvalues appearing the number of times of their multiplicity. The corresponding orthonormalized eigenstates $\psi_{i}^{0}$ satisfy the equation

$$
H^{0} \psi_{i}^{0}=E_{i}^{0} \psi_{i}^{0}, \quad\left(\psi_{i}^{0}, \psi_{j}^{0}\right)=\delta_{i j}
$$

Because $H^{0} \leqslant H$ in the sense of inequality

$$
\left(\psi, H^{0} \psi\right) \leqslant(\psi, H \psi)
$$

for every $\psi$ in the domain of $H$, it follows from the Weyl comparison theorem [15] that

$$
E_{i}^{0} \leqslant E_{i}, \quad(i=1,2, \ldots) .
$$

Therefore, the eigenvalues of $H^{0}$ already provide a rough lower bound to the eigenvalues of $H$. The Hamiltonian $H^{0}$ is called the base Hamiltonian as usual. It is worth noticing that the decomposition (5) is not unique and can be tailored to meet the requirements of a particular problem in question.

The basic idea of the method of intermediate problems is to approximate the original Hamiltonian $H$ from below by a non-decreasing sequence of the so-called truncated intermediate Hamiltonians $H^{l, k}$. These Hamiltonians are to be constructed to satisfy the inequalities

$$
\begin{aligned}
& H^{l, k} \leqslant H^{l+1, k} \leqslant H^{k} \leqslant H, \quad(l, k=1,2, \ldots), \\
& H^{l, k} \leqslant H^{l, k+1} \leqslant H, \quad(l, k=1,2, \ldots) .
\end{aligned}
$$

Therefore, the Hamiltonians $H^{l, k}$ increase whatever index $k$ or $l$ is increased and thus must give improvable lower bounds for the lowest eigenvalues of the original Hamiltonian $H$. It was shown [19] that the truncated Hamiltonians $H^{l, k}$ can be represented in a general form

$$
H^{l, k}=H^{l, 0}+H^{\prime} P^{k}, \quad(l, k=1,2, \ldots) .
$$


Here the Hamiltonian $H^{l, 0}$ is a truncation of the base Hamiltonian $H^{0}$ of the order $l$ defined as

$$
H^{l, 0} \psi=\sum_{i=1}^{l}\left(\psi_{i}^{0}, \psi\right) E_{i}^{0} \psi_{i}^{0}+E_{l+1}^{0}\left[\psi-\sum_{i=1}^{l}\left(\psi_{i}^{0}, \psi\right) \psi_{i}^{0}\right], \quad(l=1,2, \ldots),
$$

or, alternatively, in Dirac's more transparent bra and ket notation

$$
H^{l, 0}=\sum_{i=1}^{l} E_{i}^{0}\left|E_{i}^{0}\right\rangle\left\langle E_{i}^{0}\right|+E_{l+1}^{0}\left[\hat{I}-\sum_{i=1}^{l}\left|E_{i}^{0}\right\rangle\left\langle E_{i}^{0}\right|\right], \quad(l=1,2, \ldots),
$$

where $\hat{I}$ stands for the identity operator. Truncations of $H^{0}$ satisfy the inequalities

$$
H^{l, 0} \leqslant H^{l+1,0} \leqslant H^{0}, \quad(l=1,2, \ldots)
$$

which were proved in general case in [20].

The operator $P^{k}$ defines a projection of an arbitrary vector $\phi$ in the domain of $H$ onto the subspace formed by a sequence of linearly independent vectors $p_{1}, p_{2}, \ldots, p_{k}$ :

$$
P^{k} \phi=\sum_{i=1}^{k} \alpha_{i} p_{i}
$$

where constants $\alpha_{i}$ must satisfy the equations

$$
\left[p_{j}, P^{k} \phi\right]=\left[p_{j}, \phi\right]=\sum_{i=1}^{k} \alpha_{i}\left[p_{j}, p_{i}\right], \quad(j=1,2, \ldots, k) .
$$

Here an auxiliary inner product with respect to the metric operator $H^{\prime}$ was introduced as

$$
[\psi, \phi]=\left(\psi, H^{\prime} \phi\right)=\int \psi^{*} H^{\prime} \phi \mathrm{d} \tau
$$

for every pair of vectors $\psi, \phi$ for which $H^{\prime} \psi$ and $H^{\prime} \phi$ are defined. These vectors $p_{i}$ are to be normalizable in the sense of the original inner product $(6)$, i. e.

$$
\left(p_{i}, p_{i}\right)=\mathcal{N}_{i}, \quad \mathcal{N}_{i}<+\infty, \quad(i=1,2, \ldots, k)
$$

but neither their explicit normalization nor orthonormalization are required.

Projections $P^{k}$ become larger with the increase of the number $k$ of the elements $p_{i}$ involved. As a consequence, the following inequality holds

$$
0 \leqslant\left[\phi, P^{k} \phi\right] \leqslant\left[\phi, P^{k+1} \phi\right] \leqslant[\phi, \phi], \quad(k=1,2, \ldots),
$$

which in terms of original inner product reads as

$$
0 \leqslant\left(\phi, H^{\prime} P^{k} \phi\right) \leqslant\left(\phi, H^{\prime} P^{k+1} \phi\right) \leqslant\left(\phi, H^{\prime} \phi\right), \quad(k=1,2, \ldots) .
$$

From equations (12), (13) it follows that

$$
H^{\prime} P^{k} \phi=\sum_{i, j=1}^{k}\left(H^{\prime} p_{i}, \phi\right) b_{i j} H^{\prime} p_{j}
$$

where $b_{i j}$ are the elements of the matrix inverse to the matrix with terms $\left[p_{j}, p_{i}\right]$. As a consequence of equation (14)

$$
H^{\prime} P^{k} \leqslant H^{\prime} P^{k+1} \leqslant H^{\prime}, \quad(k=1,2, \ldots)
$$


and the intermediate truncated Hamiltonians $H^{k}$ defined as

$$
H^{k}=H^{0}+H^{\prime} P^{k}, \quad(k=1,2, \ldots)
$$

satisfy inequalities

$$
H^{k} \leqslant H^{k+1} \leqslant H
$$

by construction if inequalities (15) are taken into account. According to equations (11) and (15)

$$
H^{l, k} \leqslant H^{l, k+1} \leqslant H^{l, 0}+H^{\prime} \leqslant H, \quad(l, k=1,2, \ldots) .
$$

Therefore, the lowest ordered eigenvalues $E_{i}^{l, k}$ of $H^{l, k}$ must satisfy the parallel inequalities

$$
E_{i}^{l, k} \leqslant E_{i}^{l+1, k} \leqslant E_{i}^{k} \leqslant E_{i}, \quad(i, l, k=1,2, \ldots),
$$

and

$$
E_{i}^{l, k} \leqslant E_{i}^{l, k+1} \leqslant E_{i}, \quad(i, l, k=1,2, \ldots),
$$

thus providing improvable lower bounds for the original eigenvalues $E_{i}$ of the Hamiltonian $H$.

As was proved in [20], the so-constructed Hamiltonian $H^{l, k}$ can have no continuous spectrum and must have $E_{l+1}^{0}$ as an eigenvalue of infinite multiplicity. Therefore, only those eigenvalues of $H^{l, k}$ that are smaller or equal to $E_{l+1}^{0}$ can be considered as lower bound estimates for the eigenvalues of the initial Hamiltonian $H$.

The truncation procedure (10) can be significantly improved from the point of view of practical calculations if the original Hamiltonian $H$ is formally decomposed as

$$
H=H^{l, 0}+\left(H^{0}-H^{l, 0}\right)+H^{\prime}=H^{l, 0}+H^{\prime}+H^{\prime \prime}=H^{l, 0}+\tilde{H}^{\prime}, \quad(l=1,2, \ldots),
$$

where the difference $\tilde{H}^{\prime}=H-H^{l, 0}$ is obviously positive and can play the role played before by the metric operator $H^{\prime}$. In this case the positive contributions from the operator

$$
H^{\prime \prime}=H^{0}-H^{l, 0}, \quad(l=1,2, \ldots)
$$

to lower bound estimates are not simply neglected at will but rather carefully taken into consideration on common grounds with the contributions stemming from $H^{\prime}$, thus making these bounds higher than they might have been otherwise under the original truncation procedure (10).

The eigenvalues and eigenstates of the intermediate Hamiltonians $H^{l, k}$ of any order (i. e. for arbitrary magnitudes of the indices $l$ and/or $k$ ) can be expressed analytically or calculated numerically in terms of the known eigenvalues and eigenstates of $H^{0}$ and an arbitrarily chosen set of linearly independent vectors $p_{i},(i=1, \ldots, k)$. It was proved in [19] that those eigenvalues of the Hamiltonian (9) different from $E_{l+1}^{0}$ (and also from $E_{1}^{0}, \ldots, E_{l}^{0}$, should there be any eigenvalues of this kind in some special cases ) are the roots of the equation

$\operatorname{det}\left\{\left(p_{j}, H^{\prime} p_{i}\right)+\sum_{\nu=1}^{l} \frac{\left(\psi_{\nu}^{0}, H^{\prime} p_{i}\right)\left(H^{\prime} p_{j}, \psi_{\nu}^{0}\right)}{E_{\nu}^{0}-E}+\frac{\left(H^{\prime} p_{j}, H^{\prime} p_{i}\right)-\sum_{\nu=1}^{l}\left(\psi_{\nu}^{0}, H^{\prime} p_{i}\right)\left(H^{\prime} p_{j}, \psi_{\nu}^{0}\right)}{E_{l+1}^{0}-E}\right\}=0$

Each solution of (18) provides $n$ linear independent eigenfunctions of $H^{l, k}$ where $n$ is the nullity of the coefficient matrix in (18). If the number of such eigenfunctions is less than $k+l$ then it is necessary to verify if some of the eigenvalues $E_{1}^{0}, E_{2}^{0}, \ldots, E_{l}^{0}$ of $H^{l, 0}$ are also the eigenvalues of $H^{l, k}$. A verification algorithm for the test of this assumption was outlined in [19].

From the consideration above it is clear that $H^{l, k}$ may possess at most $l+k$ eigenvalues different from $E_{l+1}^{0}$. Let us notice that $E_{l+1}^{0}$ is an eigenvalue of $H^{l, k}$ of infinite multiplicity by construction and each of its corresponding eigenfunctions is orthogonal to all other eigenfunctions of the Hamiltonian (9). To obtain the lower bounds for the eigenvalues $E_{i}$ of the original Hamiltonian 
$H$, the so-obtained eigenvalues of $H^{l, k}$ lying below $E_{l+1}^{0}$ are to be ordered in a nondecreasing sequence

$$
E_{1}^{l, k} \leqslant E_{2}^{l, k} \leqslant \cdots \leqslant E_{t}^{l, k}, \quad t \leqslant l+k,
$$

in which each eigenvalue is repeated according to its multiplicity. Then the lower bounds result from the fundamental inequalities (16) and (17) as

$$
E_{i}^{l, k} \leqslant E_{i}, \quad(i=1,2, \ldots, t), \quad E_{l+1}^{0} \leqslant E_{i}, \quad(i=t+1, t+2, \ldots) .
$$

\section{Intermediate problems for the Fröhlich polaron model}

For the particular Hamiltonian (2) the Hamiltonians $H_{0}$ and $H^{\prime}$ in (5) can be identified with

$$
H_{0}=\sum_{\mathbf{k}} a_{\mathbf{k}}^{+} a_{\mathbf{k}}-\sum_{\mathbf{k}}|V(k)|^{2}-\varepsilon
$$

and

$$
H^{\prime}=\frac{1}{2}\left(\mathbf{P}-\sum_{\mathbf{k}} \mathbf{k}\left(a_{\mathbf{k}}^{+}-V(k)\right)\left(a_{\mathbf{k}}-V^{*}(k)\right)\right)^{2}+\varepsilon,
$$

respectively. Here an arbitrary positive parameter $\varepsilon$ was introduced formally and identically to ensure strict positivity of the Hamiltonian $H^{\prime}$ as required by the positivity condition (7). Later this parameter can be chosen arbitrarily small or may even be employed appropriately as a variational parameter. Because by construction $\tilde{\tilde{H}}=H_{0}+H^{\prime} \quad \forall \varepsilon$, then $E_{\mathrm{g}} \geqslant E_{\mathrm{g}}^{0}+E_{\mathrm{g}}^{\prime}$, where $E_{\mathrm{g}}, E_{\mathrm{g}}^{0}$ and $E_{\mathrm{g}}^{\prime}$ are the ground-state energies of $\tilde{\tilde{H}}, H_{0}$ and $H^{\prime}$ respectively. Since $E_{\mathrm{g}}^{\prime}=\varepsilon$, we have

$$
E_{\mathrm{g}} \geqslant-\sum_{\mathbf{k}}|V(k)|^{2} \rightarrow-\alpha \frac{\sqrt{2} k_{\mathrm{D}}}{\pi} \quad \text { as } \quad V \rightarrow \infty .
$$

Inequality (23) clearly shows the above-mentioned difference between the so-called "physical" and "field-theoretical" polaron models. For the former, the ground-state energy can only decrease no faster than linearly in $\alpha$ for any fixed $k_{\mathrm{D}}$ while the latter, as is well-known, allows for the asymptotic behavior quadratic in the electron-phonon interaction constant, e.g.

$$
E_{\mathrm{g}} \leqslant-\frac{\alpha^{2}}{3 \pi},
$$

incompatible with (23) for large enough $\alpha$. The eigenvalues of $H_{0}$ are equidistant

$$
E_{1}^{0}=-\varepsilon-\sum_{\mathbf{k}}|V(k)|^{2}, \quad E_{2}^{0}=1-\varepsilon-\sum_{\mathbf{k}}|V(k)|^{2}, \ldots, \quad E_{n}^{0}=(n-1)-\varepsilon-\sum_{\mathbf{k}}|V(k)|^{2},
$$

and highly degenerate except for the ground state, which is the phonon vacuum state $|0\rangle$, but the multiplicity of all of them remains finite until the limiting transition $V \rightarrow \infty$ is carried out. The corresponding normalized eigenstates of $H_{0}$ are

$$
\begin{gathered}
\psi_{1}=|0\rangle, \quad \psi_{2}(\mathbf{k})=a_{\mathbf{k}}^{+}|0\rangle \equiv|\mathbf{k}\rangle, \ldots \\
b g \psi_{n}\left(\mathbf{k}_{1}, \ldots, \mathbf{k}_{n-1}\right) \equiv\left|\mathbf{k}_{1}, \ldots, \mathbf{k}_{n-1}\right\rangle \sim a_{\mathbf{k}_{1}}^{+} a_{\mathbf{k}_{2}}^{+} \ldots a_{\mathbf{k}_{n-1}}^{+}|0\rangle .
\end{gathered}
$$

These groups of eigenstates span subspaces with zero, one and $n-1$ phonons respectively. Now, let us design a sequence of the truncated Hamiltonians $H^{l, 0}$ for the polaron model. For our purposes it would be convenient to choose them as

$$
\begin{aligned}
g H^{1,0}= & E_{1}^{0}|0\rangle\langle 0|+E_{2}^{0}[\hat{I}-|0\rangle\langle 0|], \ldots, \\
H^{n, 0}= & E_{1}^{0}|0\rangle\left\langle 0\left|+\cdots+E_{n}^{0} \sum_{\mathbf{k}_{1}, \ldots, \mathbf{k}_{n-1}}^{\tilde{\mathbf{k}_{1}}}\right| \mathbf{k}_{1}, \ldots, \mathbf{k}_{n-1}\right\rangle\left\langle\mathbf{k}_{1}, \ldots, \mathbf{k}_{n-1}\right| \\
& +E_{n+1}^{0}\left[\hat{I}-|0\rangle\left\langle 0\left|-\sum_{\mathbf{k}}\right| \mathbf{k}\right\rangle\left\langle\mathbf{k}\left|-\cdots-\sum_{\mathbf{k}_{1}, \ldots, \mathbf{k}_{n-1}}^{\tilde{\mathbf{k}_{1}}}\right| \mathbf{k}_{1}, \ldots, \mathbf{k}_{n-1}\right\rangle\left\langle\mathbf{k}_{1}, \ldots, \mathbf{k}_{n-1}\right|\right],
\end{aligned}
$$


so that

$$
\tilde{\tilde{H}}=H_{0}+H^{\prime}=H^{1,0}+H_{1}^{\prime}=\cdots=H^{n, 0}+H_{n}^{\prime}
$$

where

$$
H_{n}^{\prime}=H_{0}-H^{n, 0}+\frac{1}{2}\left(\mathbf{P}-\sum_{\mathbf{k}} \mathbf{k}\left(a_{\mathbf{k}}^{+}-V(k)\right)\left(a_{\mathbf{k}}-V^{*}(k)\right)\right)^{2}+\varepsilon .
$$

Summation $\sum_{\mathbf{k}_{1}, \ldots, \mathbf{k}_{n}}^{\tilde{n}}$ in taken over the states $\left|\mathbf{k}_{1}, \ldots, \mathbf{k}_{n}\right\rangle$ in such a way that all the equivalent phonon states distinct from each other exclusively by permutation of quantum numbers $\mathbf{k}_{1}, \ldots, \mathbf{k}_{n}$ are counted only once. Because $H_{0}-H^{n, 0}$ are non-negative by construction, all the Hamiltonians (27) are positively definite. Hence, any of them may serve as a new $H^{\prime}$ in the intermediate problems formalism. Substituting our choice (24) and (27) for $H^{l, 0}$ and $H^{\prime}$ in equation (18) and taking recourse again to conventional Dirac's bra and ket notation throughout, we arrive at

$$
\begin{gathered}
\operatorname{det}\left\{\left\langle p_{j}\left|H_{1}^{\prime}\right| p_{i}\right\rangle+\frac{\left\langle 0\left|H_{1}^{\prime}\right| p_{i}\right\rangle\left\langle p_{j}\left|H_{1}^{\prime}\right| 0\right\rangle}{E_{1}^{0}-E}+\frac{\left\langle p_{j}\left|\left(H_{1}^{\prime}\right)^{2}\right| p_{i}\right\rangle-\left\langle 0\left|H_{1}^{\prime}\right| p_{i}\right\rangle\left\langle p_{j}\left|H_{1}^{\prime}\right| 0\right\rangle}{E_{2}^{0}-E}\right\}=0, \\
i, j=1,2, \ldots, k .
\end{gathered}
$$

This equation is a source of multitudinous lower bounds to the ground state energy of $\tilde{\tilde{H}}$ lying below the onset of the continuous spectrum. The proximity of these lower bounds to the true value is stipulated by the number of the trial states $\left\{\left|p_{i}\right\rangle, i=1, \ldots, k\right\}$ and by the particular choice of these states. At the same time, for any number and choice of the trial states the resulting equation (28) will always be polynomial in $E$. It is very important to stress that neither part of the original Hamiltonian (3) has been omitted so far upon deriving the principle equation (28) and no other approximations have been made. As a consequence, the precision of the lower bounds (28) depends totally on the particular choice of the states $\left\{\left|p_{i}\right\rangle, i=1, \ldots, k\right\}$ and the size of this set of states. The larger the size $k$ of the set, the larger the subspace spanned by this set would be providing that $\left\{\left|p_{i}\right\rangle, i=1, \ldots, k\right\}$ are linearly independent. Of course, it is worth noticing that the problem of convergence of the lower bounds to the precise values of the discrete spectrum has not been solved yet for the method of intermediate problems in general though some important encouraging partial results have been obtained for some classes of self-adjoint linear operators on Hilbert space. Therefore, it is only natural to believe that for large enough set of the trial states (28) would yield reliable lower bounds for the polaron ground state energy. Anyway, it is already seen from the formalism outlined above that the method of intermediate problems delivers improvable lower bounds and, regarding the choice of the states $\left\{\left|p_{i}\right\rangle, i=1, \ldots, k\right\}$, seems to be flexible enough to achieve sufficiently good for all practical purposes lower bounds already for a set of trial states of moderate size $k$. As to the particular choice of these trial states, it is nearly obvious even from the purely technical point of view that the natural choice for the first trial state $\left|p_{1}\right\rangle$ would be

$$
\left|p_{1}\right\rangle=|0\rangle
$$

so that equation $(28)$ reads

$$
\left\langle 0\left|H^{\prime}\right| 0\right\rangle+\frac{\left\langle 0\left|H^{\prime}\right| 0\right\rangle\left\langle 0\left|H^{\prime}\right| 0\right\rangle}{E_{1}^{0}-E}+\frac{\left\langle 0\left|\left(H^{\prime}\right)^{2}\right| 0\right\rangle-\left\langle 0\left|H^{\prime}\right| 0\right\rangle\left\langle 0\left|H^{\prime}\right| 0\right\rangle}{E_{2}^{0}-E}=0 .
$$

Despite simplicity of this equation, the stemming from its lower bound improves the trivial lower bound (23) by default due to the contribution from the positive definite operator (22) exclusively. The rest $H_{0}-H^{1,0}$ of the operator (27) cannot contribute to (30) at all because, in general case,

$$
\left(H_{0}-H^{n, 0}\right)\left|\mathbf{k}_{1}, \ldots, \mathbf{k}_{m}\right\rangle=0, \quad \text { if } \quad m \leqslant n .
$$

Also, it is easy to see that

$$
\left\langle 0\left|H_{n}^{\prime}\right| \mathbf{k}_{1}, \ldots, \mathbf{k}_{m}\right\rangle=\left\langle 0\left|H^{\prime}\right| \mathbf{k}_{1}, \ldots, \mathbf{k}_{m}\right\rangle \forall m \quad \text { and } \quad\left\langle 0\left|H^{\prime}\right| \mathbf{k}_{1}, \ldots, \mathbf{k}_{m}\right\rangle=0 \quad \text { for } \quad m \geqslant 3 .
$$


Therefore, for the $n$-th order partitioning (26) of the original Hamiltonian starting from $n \geqslant 3$ and the choice (29),

$$
\begin{aligned}
& \operatorname{det}\left\{\left\langle 0\left|H^{\prime}\right| 0\right\rangle+\frac{\left\langle 0\left|H^{\prime}\right| 0\right\rangle\left\langle 0\left|H^{\prime}\right| 0\right\rangle}{E_{1}^{0}-E}+\frac{\sum_{\mathbf{k}}\left\langle\mathbf{k}\left|H^{\prime}\right| 0\right\rangle\left\langle 0\left|H^{\prime}\right| \mathbf{k}\right\rangle}{E_{2}^{0}-E}+\frac{\sum_{\mathbf{k}_{1}, \mathbf{k}_{2}}\left\langle\mathbf{k}_{1}, \mathbf{k}_{2}\left|H^{\prime}\right| 0\right\rangle\left\langle 0\left|H^{\prime}\right| \mathbf{k}_{1}, \mathbf{k}_{2}\right\rangle}{E_{3}^{0}-E}\right. \\
& \left.+\frac{\left\langle 0\left|\left(H^{\prime}\right)^{2}\right| 0\right\rangle-\left\langle 0\left|H^{\prime}\right| 0\right\rangle\left\langle 0\left|H^{\prime}\right| 0\right\rangle-\sum_{\mathbf{k}}\left\langle\mathbf{k}\left|H^{\prime}\right| 0\right\rangle\left\langle 0\left|H^{\prime}\right| \mathbf{k}\right\rangle-\sum_{\mathbf{k}_{1}, \mathbf{k}_{2}}\left\langle\mathbf{k}_{1}, \mathbf{k}_{2}\left|H^{\prime}\right| 0\right\rangle\left\langle 0\left|H^{\prime}\right| \mathbf{k}_{1}, \mathbf{k}_{2}\right\rangle}{E_{n+1}^{0}-E}\right\}=0 .
\end{aligned}
$$

The last term in (31) can be omitted in the limit $n \rightarrow \infty, E_{n+1} \rightarrow \infty$, and the resulting equation provides the best lower bound available for the particular choice (29) of $\left|p_{1}\right\rangle$.

It is also seen from the structure of (18) that, in general, neither particular choice of only one single trial state $\left|p_{1}\right\rangle$ can produce lower bound to the ground state energy higher than the second eigenvalue $E_{2}^{0}$ of the Hamiltonian $H_{0}$ unless, of course, $\left\langle 0\left|H^{\prime}\right| p_{1}\right\rangle=0$. To obtain the higher lying (and thus better) lower bounds one has to extend the set of trial states $\left\{\left|p_{i}\right\rangle\right\}$ and, consequently, to enlarge the subspace on which $H^{\prime}$ is projected. In principle, there are many ways of choosing these additional trial functions but it would be prudent to choose them so as to ensure explicit analytical calculability of all matrix elements in equation (18) first of all. Then, it would be desirable to project $H^{\prime}$ onto the subspace formed by those trial states which have already been used in polaron studies successfully, for example, the states employed in variational approaches aimed at derivation of the best available upper bounds to the polaron energy spectrum. Among them, the Lee-Law-Pines variational function

$$
\left|\psi_{\mathrm{LLP}}^{(0)}\right\rangle=U^{-1} \cdot U_{\mathrm{LLP}}\left(f^{(0)}(\mathbf{k})\right)|0\rangle=U^{-1} \cdot \exp \left\{\sum_{\mathbf{k}}\left(a_{\mathbf{k}}^{+} f^{(0)}(\mathbf{k})-a_{\mathbf{k}} f^{(0) *}(\mathbf{k})\right)\right\}|0\rangle,
$$

introduced in [14], meets these requirements and can be incorporated into the set of trial states as $\left|p_{2}\right\rangle$. Here the function $f^{(0)}(\mathbf{k})$ might subsequently be chosen to maximize the lower bound (18), which task seems to be more complicated than the one of the upper bound minimization undertaken in [14]. Or, alternatively, the value of $f(\mathbf{k})$ can be taken exactly the same as in [14]. In the same fashion, generalized Lee-Law-Pines variational states of the type

$$
\begin{gathered}
\left|\psi_{\mathrm{LLP}}^{(1)}\right\rangle=U^{-1} \cdot U_{\mathrm{LLP}}\left(f^{(1)}(\mathbf{k})\right)\left(d_{0}^{(1)}|0\rangle+\sum_{\mathbf{k}} d_{1}^{(1)}(\mathbf{k})|\mathbf{k}\rangle\right), \ldots, \\
\left|\psi_{\mathrm{LLP}}^{(n)}\right\rangle=U^{-1} \cdot U_{\mathrm{LLP}}\left(f^{(n)}(\mathbf{k})\right)\left(d_{0}^{(n)}|0\rangle+\sum_{\mathbf{k}} d_{1}^{(n)}(\mathbf{k})|\mathbf{k}\rangle+\ldots+\sum_{\mathbf{k}_{1}, \ldots, \mathbf{k}_{n}}^{\sim} d_{n}^{(n)}\left(\mathbf{k}_{1}, \ldots, \mathbf{k}_{n}\right)\left|\mathbf{k}_{1}, \ldots, \mathbf{k}_{n}\right\rangle\right),
\end{gathered}
$$

introduced by D.M. Larsen [13], can be employed as $\left|p_{3}\right\rangle,\left|p_{4}\right\rangle, \ldots,\left|p_{n+2}\right\rangle$ trial states in (18) in order to increase the dimensionality of the trial subspace.

\section{Summary}

It was shown that there are many ways of applying the method of intermediate problems to the investigation of the physical Fröhlich polaron model for which $k_{\mathrm{D}}$ is finite. Each way relies on its own choice of the original Hamiltonian partitioning (26), complemented with the customized choice of trial variational states $\left\{\left|p_{i}\right\rangle, i=1, \ldots, k\right\}$, and, therefore, yields its own sequence of improvable lower bounds to the ground state energy of the polaron at rest as well as to the adjacent low-lying 
branch of the excitation energy spectral curve of the moving polaron. Convergence of the thus obtained lower bounds to the respective true values has not been proved in general yet and, at the present stage, can be judged upon in the course of practical numerical calculations only. The sets $\left\{\left|p_{i}\right\rangle, i=1, \ldots, k\right\}$ can be chosen to make analytical calculation of all requisite matrix elements feasible. At the same time, any realistic calculations will inadvertently involve a lot of routine work. Because of this, the choice (32) for the variational states looks especially attractive as it allows for relatively easy implementation of the matrix element calculation algorithm by means of contemporary programming tools for analytical computation such as Mathematica or Maple.

It is essential to stress once again here that the method of intermediate problems, as it was presented above, was shown to be applicable to the so-called "physical" polaron model where $k_{\mathrm{D}}$ is finite, which property has been employed consistently throughout all calculations. Nevertheless, this does not mean at all that no generalization of the method to the case of the "field-theoretical" polaron model is possible. Actually, the main formal obstacle to such a generalization stems from the fact that Hamiltonian (21) becomes unbounded from below for $k_{\mathrm{D}} \rightarrow \infty$. In other words, in the partitioning (21), (22) of the original Hamiltonian (3) the Hamiltonian (22) is "too positive" whilst the Hamiltonian (21) is, in some sense, "not positive enough", which makes the whole partitioning too unbalanced for successful application of the method in the limiting case $k_{\mathrm{D}} \rightarrow \infty$. Fortunately, much more balanced, albeit not so simple, partitioning schemes exist for the Fröhlich polaron model, for example, the partitionings derived in $[11,12]$, which surely allow for treatment of the "field-theoretical" polaron model. The analysis of this and other balanced partitioning schemes with respect to the method of intermediate problems will be presented somewhere else later. Moreover, the particular approach to the intermediate problem construction employed in this study is not unique. Other implementations of the general idea of the method of intermediate problems may happen to be free from deficiencies of the presented approach and better suited for the investigation of the "field-theoretical" polaron model.

The method outlined here is in no way limited to polaron studies. Actually, it might be applied to many similar quantum models employed in solid state physics provided that these models possess a gap in the spectrum of the free field in the free Hamiltonian $H_{0}$ like it was the case here with the free phonon field. It is also desirable for the rest part of $H_{0}$, related to the free "matter" part of the model, to possess at least several discrete energy states lying below the onset of the respective continuous spectrum of the "matter" part. If this is not the case, a canonical transformation, eliminating the "matter" variables from the "field-matter" interaction in similarity to (3), should exist thus making the approach applicable.

\section{Acknowledgements}

This work is supported by the RAS research program "Fundamental Problems of Nonlinear Dynamics" and by the RFBR grant No. 09-01-00086-a. 


\title{
References
}

1. Landau L.D., Phys. Z. Sowietunion, 1933, 3, 664. [English translation: Collected Papers. Gordon and Breach, New York, p. 67-68, 1965].

2. Pekar S.I., Sov. Phys. JETP, 1946, 16, 341; Pekar S.I. Research in Electron Theory of Crystals. Gostekhizdat, Moscow, 1951. [English translation: Research in Electron Theory of Crystals. US AEC Report AEC-tr-5575].

3. Landau L.D., Pekar S.I., Sov. Phys. JETP, 1948, 18, 419.

4. Fröhlich H., Pelzer H., Zienau S., Philos. Mag., 1950, 41, 221.

5. Fröhlich H., Proc. Roy. Soc. A, 1937, 160, 230-241.

6. Fröhlich H., Advan. Phys., 1954, 3, 325-361.

7. Davydov A.S. Solid State Theory. Nauka, Moscow, p. 246-278, 1976.

8. Feynman R.P., Phys. Rev., 1955, 97, 660-665; Feynman R.P. Statistical Mechanics. Benjamin, Reading, MA, 1972

9. Bogolyubov N.N., Ukrainian Math. J., 1950, II, No. 2, 3-24.

10. Bogolyubov N.N., Bogolyubov N.N., Jr. Aspects of the Polaron Theory - In: JINR Communications P-17-81-65. Dubna, 1981; Bogolyubov N.N., Bogolyubov N.N., Jr. Aspects of Polaron Theory. Equilibrium and Nonequilibrium Problems. World Scientific Publishing Company, New Jersey, London, Singapore, Beijing, Shanghai, Hong Kong, Taippei, Chennai, 2008.

11. Lieb E.H., Yamazaki K., Phys. Rev., 1958, 111, 728-733.

12. Lieb E.H., Thomas L.E., Comm. Math. Phys., 1997, 183, 511-519; Lieb E.H., Thomas L.E., Comm. Math. Phys., 1997, 188, 499-500.

13. Larsen D.M., Phys. Rev., 1968, 172, 967-971.

14. Lee T.D., Low F., Pines D., Phys. Rev., 1953, 90, 297-302.

15. Weyl H., Bull. Am. Math. Soc., 1950, 56, 115-139.

16. Weinstein A. Etude des spectres des equations aux derivees partielles - In: Memorial des Sci. Math. No. 88, Paris, 1937.

17. Weinstein A., Stenger W. Intermediate Problems for Eigenvalues. Academic Press, New York and London, 1972.

18. Gould S.H. Variational Methods for Eigenvalue Problems: An Introduction to the Weinstein Method of Intermediate Problems, 2nd ed. Univ. of Toronto Press, 1966.

19. Bazley N.W., Fox D.W., Phys. Rev., 1961, 124, 483-491.

20. Bazley N.W., Fox D.W., J. Research Natl. Bur. of Standards B, 1961, 65, No. 2, 105-111.

\section{Метод проміжних задач у моделі полярона Фрьоліха}

\section{В.А. Солдатов}

Математичний інститут ім. В.А. Стєклова, вул. Губкіна 8, Москва 119991, Росія

Отримано 13 червня 2009 р., в остаточному вигляді - 14 липня 2009 р.

\begin{abstract}
Метод проміжних задач в теорії лінійних напівобмежених самоспряжених операторів у пристосованому просторі Гільберта використовується для дослідження енергії основного стану в моделі полярона Фрьоліха. Показано, що для довільних значень константи електрон-фононної взаємодії можуть бути отримані різні безмежні неспадні послідовності оцінок знизу для енергії основного стану полярона. Запропонований підхід дозволяє отримати точні числові оцінки знизу у всіх порядках і може бути узагальнений для вивчення нижньої гілки енергетичного спектру збуджених станів повільного полярона, що межує з енергією основного стану нерухомого полярона. В комбінації з іншими добре відомими підходами до оцінки зверху залежностей енергетичного спектру полярона Фрьоліха від константи електрон-фононної взаємодії і повного імпульсу полярона вищезгадані оцінки з уточненням знизу можуть забезпечити знаходження практично точних значень для енергії полярона, що повільно рухається.
\end{abstract}

Ключові слова: полярон, модель полярона Фрьоліха, оцінки знизу, метод проміжних задач, енергія основного стану

PACS: $71.38 .-k, 71.38 . F p$ 
